\title{
Self-Disclosure among Men and Women of Arab Descent: Implications for Group-Based Health Education
}

\author{
Elizabeth A. Bertran ${ }^{1}$, Nicole R. Pinelli ${ }^{2}$, Dana El Masri ${ }^{1}$, Stephen J. Sills ${ }^{3}$, Linda Jaber ${ }^{1{ }^{1 *}}$ \\ ${ }^{1}$ Department of Pharmacy Practice, Eugene Applebaum College of Pharmacy and Health Sciences, Detroit, USA \\ ${ }^{2}$ Division of Practice Advancement and Clinical Education, University of North Carolina Eshelman School of Pharmacy, \\ Chapel Hill, USA \\ ${ }^{3}$ Department of Sociology, University of North Carolina at Greensboro, Greensboro, USA \\ *Corresponding author: ljaber@wayne.edu
}

\begin{abstract}
Arab American gender norms may affect female participation in group-delivered health education settings. Our objective was to examine gender-specific participation in Arab American group interactions. This study was conducted to inform the necessity of gender-specific groups in a subsequent diabetes prevention intervention. Self-identified Arabs or Arab Americans $\geq 30$ years and without diabetes were randomly recruited. Participants were randomly assigned to male-only, female-only, or mixed-gender focus groups. A trained Arabic-speaking moderator facilitated 90-minute sessions using a standardized guide. Interviews were digitally recorded and transcribed verbatim. A self-disclosure survey immediately followed sessions. Turn-taking, verbosity, and survey responses were compared between males and females in gender-specific and mixed-gender group settings. Twenty-nine individuals participated: male-only (8), female-only (12), and mixed-gender (9). Males took more turns and spoke with more utterances than females during gender-specific and mixed-gender groups. Fewer men reported keeping comments to themselves relative to women. Group-formatted educational interventions in Arab Americans should take self-disclosure into consideration and efforts should be made to identify females who prefer participating in a gender-specific group.
\end{abstract}

Keywords: patient education, health education, cultural diversity, community health, minority populations, selfdisclosure

Cite This Article: Elizabeth A. Bertran, Nicole R. Pinelli, Dana El Masri, Stephen J. Sills, and Linda Jaber, "Self-Disclosure among Men and Women of Arab Descent: Implications for Group-Based Health Education." American Journal of Educational Research, vol. 6, no. 3 (2018): 196-200. doi: 10.12691/education-6-3-6.

\section{Introduction}

Culture has a significant effect on how an individual seeks and receives health care [1,2,3]. Social norms and mores can delineate when, where, how and to whom health care is delivered, thus a generic approach to health interventions might not be appropriate for all cultures [4]. The growing Arab American population bears a significant burden of chronic diseases, including diabetes, metabolic syndrome, and hypertension [5,6,7,8,9]. However, there are a few educational and lifestyle interventions to address these diseases that take into consideration the effect of Arab culture on healthcare delivery.

Certain aspects of Arab American culture might have significant implications on health interventions. These effects are more pronounced among the more conservative Arabs where women have restrictions on how and with whom they may interact. Men are expected to speak on behalf of the women in their family [3], thus women have less prerogative on communications with non-relatives. This can be especially critical when a stigmatized disease (e.g. comorbid depression with chronic illness) can reflect poorly on the reputation of the family, which is closely guarded in Arab culture [3]. A relevant example of this cultural norm in health care is the inclusion and participation of a woman's family in her medical appointments [3]. The collectivist nature of Arab culture also implicates a tendency for consensus in Arab group discussions which would highlight corporate over individual views [10]. A woman may feel that her individual thoughts are not important in light of the opinions of her community and avoid disclosure of her personal views, especially if they are contrary to the views of her male relatives. Collective consideration of these cultural factors suggest that the views of an Arab woman are potentially filtered by her male relatives and modified by community consensus.

These cultural factors are clearly implicated in educational health interventions for Arab Americans. Health programs often utilize group-formatted curricula [11], which would likely appeal to Arab Americans since it would leverage the Arab cultural emphasis on social interaction. However, given the cultural factors identified above, program developers might be concerned that women may be "silenced" in the presence of non-relatives, especially males, or avoid self-disclosure in mixed-gender group settings to encourage consensus; this phenomenon has not yet been documented in this population. The 
objective of this study was to systematically examine the degree of silencing and lack of self-disclosure among Arab American women in mixed-gender group interactions. Our central hypothesis was that women are less comfortable and less likely to disclose information in a mixed-gender setting.

\section{Materials and Methods}

The current study preceded the initiation of a groupformatted diabetes prevention intervention in Arab Americans. While we were hosting focus groups with Arab Americans on how to culturally adapt the intervention, we analyzed group discussions for silencing or inhibition of self-disclosure. This investigation would enable us to determine the necessity of gender-specific educational groups in the subsequent intervention. The study was approved by the Wayne State University Institutional Review Board. All participants provided written informed consent.

Eligibility criteria were designed to best represent those individuals who would participate in the subsequent intervention. Self-identified Arab or Arab Americans $\geq 30$ years of age and without diabetes were identified from a previously constructed list of 542 individuals and from the general public in Dearborn, Michigan. Eligible and interested individuals that were willing to be randomized were telephoned by the study coordinator and invited to participate in a 90-minute focus group session conducted between November and December 2007. Participants were randomly assigned to a male-only, female-only, or mixed-gender focus group.

All three focus groups were conducted at a clinic conference room at the Arab Community Center for Economic and Social Services in Dearborn, Michigan, a health and social service community center that primarily serves Arab Americans. All sessions were conducted in Arabic and facilitated by an Arabic-fluent female moderator who had been trained by an expert qualitative methodologist using the interview guide developed by the study team. The guide included questions that were focused on identifying salient cultural beliefs on diabetes prevention to inform development of our subsequent intervention. The moderator covered each topic systematically and completely; all participants were encouraged to express themselves on all topics. Two additional staff members were present acting as note-takers and operating recording equipment. Interviews were digitally recorded (Sony ICD-ST25s), transcribed verbatim, and translated into English by a professional translator. A survey was administered immediately following the group sessions to all participants to collect demographic information and to assess self-disclosure during the discussions. A 5-point Likert scale (where $5=$ strongly agree and $1=$ strongly disagree) was used to measure the extent to which participants agreed with statements related to their participation and comfort in the focus group sessions.

Data from focus group discussions were imported into MAXQDA, a data management program. Discourse analysis $[12,13,14]$ was applied to transcripts to measure turn-taking (number of turns taken by gender) and verbosity (character, word, line and paragraph counts averaged per person per minute). Post-session survey responses were coded and entered into a spreadsheet; descriptive statistics were conducted using SPSS version 21 (Armonk, New York: IBM Corp). Data are presented as median \pm (interquartile range) IQR for continuous variables and percentages for categorical variables, unless otherwise noted.

\section{Results}

Twenty-nine individuals (62\% female) participated in the study and were fairly representative of the broader Arab American community. As expected, the largest proportion of participants were from Lebanon $(\mathrm{N}=10)$, followed by Syria $(\mathrm{N}=4)$, Palestine $(\mathrm{N}=4)$, and Yemen $(\mathrm{N}=4)$. Additional participants represented other Arab national origins: Egypt, Jordan, and Iraq. Only one participant was born in the US. Mean age was similar between male and female participants (42.0 vs. 41.5 years), respectively. Residence time in the US ranged from "only a few months" to "more than 37 years" and most participants had immigrated to the US less than 10 years ago $(\mathrm{N}=17)$. Most women reported being homemakers.

Group size ranged from eight to twelve participants and sessions were 90 to 115 minutes in duration. Three tests of silencing were measured: turn-taking, verbosity, and selfreported measures of self-disclosure.

Table 1. Analysis of Turn-Taking by Participants

\begin{tabular}{|c|c|c|c|}
\hline Participant & Male-only & Female-only & Mixed gender \\
\hline Man 1 & 15 & & \\
\hline Man 2 & 26 & & \\
\hline Man 3 & 36 & & \\
\hline Man 4 & 18 & & \\
\hline Man 5 & 37 & & \\
\hline Man 6 & 15 & & \\
\hline Man 7 & 51 & & \\
\hline Man 8 & 34 & & \\
\hline Woman 1 & & 35 & \\
\hline Woman 2 & & 18 & \\
\hline Woman 3 & & 37 & \\
\hline Woman 4 & & 33 & \\
\hline Woman 5 & & 22 & \\
\hline Woman 6 & & 20 & \\
\hline Woman 7 & & 21 & \\
\hline Woman 8 & & 32 & \\
\hline Woman 9 & & 13 & \\
\hline Woman 10 & & 6 & \\
\hline Woman 11 & & 23 & \\
\hline Woman 12 & & 6 & \\
\hline Man 9 & & & 31 \\
\hline Man 10 & & & 38 \\
\hline Man 11 & & & 53 \\
\hline Woman 13 & & & 13 \\
\hline Woman 14 & & & 33 \\
\hline Woman 15 & & & 36 \\
\hline Woman 16 & & & 15 \\
\hline Woman 17 & & & 12 \\
\hline Woman 18 & & & 8 \\
\hline $\begin{array}{l}\text { Median } \\
(\mathrm{IQR})^{\mathrm{a}}\end{array}$ & $30(15.8,36.8)$ & $21.5(14.3,32.8)$ & $\begin{array}{c}31 \text { (12.5, } 37.0), 38 \\
\text { (Male), } 14 \text { (female) }\end{array}$ \\
\hline
\end{tabular}

${ }^{\mathrm{a}} \mathrm{IQR}=$ interquartile range. 
Table 2. Analysis of Text Units by Group

\begin{tabular}{|l|c|c|c|c|c|c|c|c|c|}
\hline Condition & $\mathrm{N}$ & $\begin{array}{c}\text { Time } \\
\text { (minutes) }\end{array}$ & Words & Characters & Paragraphs & Lines & $\begin{array}{c}\text { Words per } \\
\text { minute }\end{array}$ & $\begin{array}{c}\text { Words per } \\
\text { person }\end{array}$ & $\begin{array}{c}\text { Words per person per } \\
\text { minute }\end{array}$ \\
\hline Men & 8 & 90 & 6,869 & 31,902 & 320 & 712 & 76 & 859 & 10 \\
\hline Women & 12 & 115 & 8,952 & 41,620 & 351 & 767 & 78 & 746 & 6 \\
\hline Mixed & & 100 & & & & & & & \\
\hline Men & 3 & & 3,992 & 18,269 & 92 & 290 & 40 & 1331 & 13 \\
\hline Women & 6 & & 3,478 & 15,428 & 128 & 271 & 35 & 580 & 6 \\
\hline
\end{tabular}

Table 3. Post-focus Group Silencing Survey Measures

\begin{tabular}{|c|c|c|c|c|}
\hline \multirow{3}{*}{ Question } & \multicolumn{4}{|c|}{ Percent Somewhat or Strongly Agree $^{a}$} \\
\hline & \multirow{2}{*}{ Male $(\mathrm{N}=8)^{\mathrm{b}}$} & \multirow{2}{*}{ Female $(\mathrm{N}=12)^{\mathrm{b}}$} & \multicolumn{2}{|c|}{ Mixed Gender } \\
\hline & & & Male $(\mathrm{N}=3)$ & Female $(\mathrm{N}=6)^{\mathrm{b}}$ \\
\hline I was comfortable sharing my perspective in this group setting & 100 & 100 & 100 & 100 \\
\hline I felt free to express my own opinions & $100(7 / 7)$ & 100 & 100 & 100 \\
\hline I felt others were open to what I had to say & 100 & $100(11 / 11)$ & 100 & 100 \\
\hline I had things to say which I kept to myself & $60.0(3 / 5)$ & $45.5(5 / 11)$ & 0 & 50.0 \\
\hline I felt others dominated the discussion & 50.0 & $40.0(4 / 10)$ & 0 & 16.7 \\
\hline I felt awkward sharing in front of the group & $14.3(1 / 7)$ & 16.7 & 0 & 50.0 \\
\hline There were uncomfortable moments during the focus group & 25.0 & 16.7 & 0 & 66.7 \\
\hline I was happy to participate in this research & 100 & $100(10 / 10)$ & 100 & $100(5 / 5)$ \\
\hline I could have said more than I did & $57.1(4 / 7)$ & 58.3 & 33.3 & 50.0 \\
\hline I feel more comfortable in same-sex groups & 62.5 & $72.7(8 / 11)$ & 0 & 50.0 \\
\hline I felt there were too many people in the group & 37.5 & 33.3 & 0 & 33.3 \\
\hline I will participate in a future focus group & 100 & 100 & 33.3 & 100 \\
\hline
\end{tabular}

${ }^{\mathrm{a}}$ A 5-point Likert Scale, where 1 = strongly disagree, 2 = somewhat disagree, 3 = neutral, 4 = somewhat agree, $5=$ strongly agree

${ }^{\mathrm{b}}$ Except where otherwise noted.

Analysis of turn-taking by participants is found in Table 1 . Men took more turns speaking in the male-only group compared to women in the female-only group (30 vs 21.5 turns). In the mixed-gender group, though men were the minority, they spoke more than twice as often as women (38 vs. 14 turns). Individual analysis shows that several male participants dominated the conversations in their respective focus groups. Turn-taking for women was more equitably distributed, though some women clearly took fewer turns speaking than others.

Analysis of utterances per person (verbosity) is found in Table 2. Men were more verbose than their female counterparts. Men in both the male-only as well as the mixed-gender group produced more utterances (10 vs. 6; 13 vs. 6 words per person per minute, respectively). In particular, men in the mixed-gender group contributed over twice as much even though there were half as many of them (1331 vs. 580 words per person). Women's utterances were, however, consistent in both the mixed-gender group and the female-only group.

Self-disclosure survey responses are reported in Table 3. Men were more likely to participate in the discussion compared to female participants. Women reported keeping things to themselves more often than men (41.7 vs. $37.5 \%)$. Women were also more likely to report having more to say than they did (58.3 vs. $50.0 \%$ ), feeling awkward sharing in front of the group (16.6 vs. 12.5\%), and feeling more comfortable in a same-gender group (66.7 vs. $62.5 \%$ ). When survey responses were compared by sex in the mixed-gender focus group, a similar and more pronounced female silencing trend was noted.

\section{Discussion}

The findings support our hypothesis that female participation is inhibited in mixed-gender focus group interactions, lending support to the practice of gender-specific educational groups. Measures of verbosity, turn-taking, and self-disclosure were higher in males than females. This finding was even more pronounced when comparing data by gender in the mixed-gender focus group. Speech in groups with men, tended to be dominated by fewer speakers, while the female-only group was more equitable in turn-taking and amount of discourse per participant. The reluctance to self-disclose may result in loss of important discourse relevant to women since women were less likely to talk, more likely to feel "awkward", and more likely to keep information to themselves. Moreover, women indicated that they were far more comfortable in same-sex groups.

This is the first study to document the existence of female silencing in mixed-gender focus group discussions among Arab Americans. The silencing demonstrated here has significant implications to group-based health educational interventions in this population. Women in mixed-gender educational groups might feel inhibited from sharing views or questions, thus ignoring topics especially pertinent to them. They also may acquiesce to group consensus, which was suggested in the survey responses that indicated a substantial proportion of women, regardless of group type, reported keeping things to themselves and having more to say than they did. A woman's personal experiences and questions may never 
surface, thus limiting the benefits she could receive from group education.

This phenomenon of female self-inhibition likely also applies to other health intervention components in this population, e.g. physical activities. As seen in a previous study, $20 \%$ of participants requested a same-gender lifestyle intervention group for diabetes prevention, which included exercise sessions [15]. Notably, a large majority of participants had immigrated from Lebanon where gender roles are not as strictly enforced, thus this percentage would likely be higher in a more diverse sample. Lebanon is a multicultural, cosmopolitan society and is portrayed as the most westernized Arab nation. It is often perceived as an interaction of the Western world and the Middle East [16]. Lebanon has been described as a pioneer in the Middle Eastern region promoting the participation of women in various economic and political activities [17]. Therefore, Lebanese women are seen as modern, often liberated from the veil, and interact freely with men [16]. This is in contrast to women in other Middle Eastern countries where cultural restrictions exist, often limiting their roles in society.

Findings from this study may also apply to other maledominated cultures. Patriarchy has been shown to affect female self-disclosure in a variety of populations, including countries in southeast Asia [18] and Europe [19]. This would be expected to affect group-formatted education in these populations as well. Investigators involving patriarchal cultures need to cautiously design group-formatted educational interventions to account for potential silencing. Further research is needed in patriarchal ethnic groups to determine the necessity of gender separation.

There are potential limitations of our study. First, the sample size was relatively small, and the groups were not of equal size. However, the measure (amount of text in turns, lines, or words) was reported per individual per unit time to minimize the impact of this limitation. Second, the mixed group was imbalanced, with only three males and six females, although males still participated more than females. Third, due to the pilot nature of the study, only one group per condition was conducted, however the strictly objective nature of the analysis strengthens the reliability of the data. Finally, at one point near the end of the discussion in the mixed-gender group, the facilitator informally commented on male participation. Although this interjection may have biased the results for this session, it was near the end and thus would have contaminated only the small amount of data after its occurrence. Additionally, this type of interference was not observed in the other focus group sessions.

\section{Conclusions}

Although this data makes a significant and needed contribution to the scientific literature for appropriate gender separation in group-formatted health education in Arab Americans, more research is still needed. The current study focused on the effect of male presence on female participation; however it is likely that female presence also affects the participation of men as well. Further research may explore if the content of male Arab
American speech is also affected by the presence of women, such as exploring is men censor the types of things they say in front of women. Additional research is required to further explore the dynamics of group interactions in this community. The current study provides the pilot data required to further such research in a larger sample of Arab Americans.

Our findings document the phenomenon of female silencing in mixed-gender Arab American groups. Health care providers, especially educators, should recognize female silencing while conducting their educational interventions.

Although Arab Americans value social interaction and may prefer group-conducted health education [15], female silencing can occur in mixed-gender groups. Therefore, providers would benefit from familiarization of Arab American cultural norms in order to address potentially affected components in a culturally-sensitive manner. It's also critical for providers to recognize the potential for female patients to not disclose important information about their health in this setting. Therefore, providers may consider actively identifying female concerns and ensuring that these are addressed even if not prompted by the group. An additional consideration is to have women indicate whether they have a preference for female-only or mixed-gender group discussions.

\section{Acknowledgements}

This work was supported by the National Institute of Diabetes and Digestive and Kidney Diseases [1-R34-DK076663-01A1]. EA Bertran and NR Pinelli were significantly involved in data interpretation and manuscript preparation. D El Masri was involved in data interpretation and contributed to the manuscript. SJ Sills provided guidance on methodology, statistical analysis, interpretation of findings, and contributed to the manuscript. L Jaber was involved in developing the research questions and methodology, reviewing identified references and data collection forms, interpretation of findings, and significantly contributed to manuscript writing.

\section{References}

[1] Aboul-Enein, B.H. (2010). "The cultural gap delivering health care services to Arab American populations in the United States", Journal of Cultural Diversity, 17(1), 20.

[2] Kulwicki, A. (1996) "An ethnographic study of illness perceptions and practices of Yemeni-Arabs in Michigan”, Journal of Cultural Diversity, 3(3), 80-89.

[3] Mourad, M.R. and Carolan, M.T.A. (2010). "An ecological approach to culturally sensitive intervention for Arab American women and their families”, The Family Journal, 18(2), 178-183.

[4] Halcomb, E.J., Gholizadeh. L., DiGiacomo, M., Phillips, J. and Davidson, P.M. (2007). "Literature review: considerations in undertaking focus group research with culturally and linguistically diverse groups", Journal of Clinical Nursing, 16(6), 1000-11.

[5] Dallo, F.J. and Borrell, L.N. (2006). "Self-reported diabetes and hypertension among Arab Americans in the United States", Ethnicity \& Disease, 16(3), 699-705.

[6] Dallo, F.J. and James, S.A. (2000) "Acculturation and blood pressure in a community-based sample of Chaldean-American women”, Journal of Immigrant Health, 2(3), 145-53. 
[7] Jaber, L.A., Brown, M.B., Hammad, A., Nowak, S.N., Zhu, Q., Ghafoor, A., et al. (2003). "Epidemiology of diabetes among Arab Americans”, Diabetes Care, 26(2), 308-13.

[8] Jaber, L.A., Brown, M.B., Hammad, A., Zhu, Q. and Herman, W.H. (2004). "The prevalence of metabolic syndrome among Arab Americans”, Diabetes Care, 27(1), 234-8.

[9] Jamil, H., Fakhouri, M., Dallo, F., Templin, T., Khoury, R. and Fakhouri, H. (2008). "Self-reported heart disease among Arab and Chaldean American women residing in southeast Michigan", Ethnicity \& Disease, 18(1), 19-25.

[10] Thomas, A. (2008). "Focus groups in qualitative research: culturally sensitive methodology for the Arabian Gulf?", International Journal of Research \& Method in Education, 31(1), 77-88.

[11] Warsi, A., Wang, P.S., LaValley, M.P., Avorn, J. and Solomon, D.H. (2004). "Self-management education programs in chronic disease: a systematic review and methodological critique of the literature", Archives of Internal Medicine, 164(15), 1641-9.

[12] Franzosi, R., Doyle, S., McClelland, L., Rankin, C.P. and Vicari, S. (2012). "Quantitative narrative analysis software options compared: PC-ACE and CAQDAS (ATLAS.ti, MAXqda, and NVivo)", Quality \& Quantity, 47(6), 3219-47.
[13] Liamputtong, P. (2009). "Qualitative data analysis: conceptual and practical considerations", Health Promotion Journal of Australia, 20(2), 133-9.

[14] Silver, C. and Fielding, N. (2008). "Using computer packages in qualitative research. The Sage Handbook of Qualitative Research in Psychology", 334-51.

[15] Jaber, L.A., Pinelli, N.R., Brown, M.B., Funnell, M.M., Anderson, R., Hammad, A., et al. (2011). "Feasibility of group lifestyle intervention for diabetes prevention in Arab Americans", Diabetes Research \& Clinical Practice, 91(3), 307-15.

[16] Neal, M., Finlay, J. and Tansey, R. (2005). "My father knows the minister: a comparative study of Arab women's attitudes towards leadership authority”, Women in Management Review, 20(7), 478-97.

[17] Sidani, Y. (2005). "Women, work, and Islam in Arab societies", Women in Management Review, 20(7), 498-512.

[18] Niaz, U. and Hassan, S. (2006). "Culture and mental health of women in South-East Asia”, World Psychiatry, 5(2), 118-20.

[19] Jack, D.C. and Ali, A. (2010). "Silencing the self across cultures: depression and gender in the social world", Oxford University Press. 